\title{
Health Information Work - a scoping review protocol
}

Kathleen Mary Gray ${ }^{1}$, Cecily Anne Gilbert ${ }^{1}$

${ }^{1}$ Health and Biomedical Informatics Centre, University of Melbourne, Melbourne, Victoria, Australia

Corresponding Author:

Cecily Gilbert ${ }^{1}$

University of Melbourne, Parkville, Victoria, 3010, Australia

Email address: cecilyg@unimelb.edu.au 


\title{
Health Information Work - a scoping review protocol
}

\author{
Kathleen Mary Gray ${ }^{1}$, Cecily Anne Gilbert ${ }^{1}$ \\ ${ }^{1}$ Health and Biomedical Informatics Centre, University of Melbourne, Melbourne, Victoria, \\ Australia
}

Corresponding Author:

Cecily Gilbert ${ }^{1}$

University of Melbourne, Parkville, Victoria, 3010, Australia

Email address: cecilyg@unimelb.edu.au

\begin{abstract}
Background. The work of managing health data, health information and health knowledge is a vital, yet unacknowledged, function in our current health system, especially in the era of digital health. This protocol is for a literature review which explores the evolution and development of the concept of health information work.

Methodology. A scoping review of published literature in the domains of health sciences, information technology and information sciences has been carried out. A thematic and bibliometric analysis of the resulting set of publications is currently being undertaken.

Results. The review results will shed light on the responsibilities and the contributions of the health information workforce, with a synthesis of capability or competency related themes identified in the literature, and analysis of publication year spans, prominent authors, institutions and source journals.
\end{abstract}

Conclusion. As research interest in the wide array of health information work is increasing, the way this work is reflected in the global literature and trends reported in key publications will provide useful evidence for health information workforce planning and positioning for the future.

\section{Introduction}

Why look at the concept of health information work?

The health information workforce comprises a range of people from specialised occupations who together are responsible for the systems for capturing and using health data, health information, and health knowledge, thus directly impacting the quality and safety of patient care. Yet, precise data about the health information workforce is difficult to obtain, as it is largely invisible, illdefined, unregulated and unmonitored. 
"Health information" as a specialist work domain emerged in the 20th century. Individual professions and occupations, such as medical records managers and medical librarians, arose in the early decades of the 1900s, and developed in parallel, rather than intersecting, streams. Further specialisations emerged during the latter decades of the 1900s, in particular health informatics from mid-century onward.

However more recently the field has become very fluid, due in part to technological changes that enhance collaboration between computing or IT staff and those working with health data or health information. A range of professions now claim expertise in health information work, and position titles and career paths also vary greatly. Moreover, in the current era of digital health, the health information workforce is in a dynamic situation, confronting issues of relevance and sustainability in the face of possible workforce structuring.

As the imperative strengthened to define and predict this workforce, surveys of workforce numbers and characteristics have been undertaken. In 2006, the UK National Health Service report on its informatics workforce was published (Eardley, 2006), followed by a review of the Australian health informatics workforce in 2009 (Legg \& Lovelock, 2009), and a Canadian report in the same year on its health informatics and health information management human resources (O’Grady, 2009). Hersh (2010) reviewed prior research on the health information technology workforce as part of estimating future demand, and concluded:

"There is increasing recognition that a competent and well-trained workforce is required for successful implementation of health information technology...Substantial numbers of individuals are needed with diverse competencies." (p. 204)

In 2013 the national government agency Health Workforce Australia (HWA) examined the Australian health information workforce. HWA concluded that the workforce was poorly defined, with a lack of accurate data about the professional areas that comprise the workforce. HWA recommended that the workforce should be clearly delineated, data collection should be improved, and its future configuration should be addressed - not least to deal with anticipated workforce shortfalls. Extensive research has been conducted in Australia, both prior to and following the HWA recommendations, to examine health information competencies for specialists, recognition of health information in the standard occupational classifications, and education requirements in health information for the clinical workforce.

This present literature review is part of a larger research program in Australia which is looking at the changing nature and scope of the existing health information workforce, and clarifying the roles and responsibilities of those in it. A national Health Information Workforce Census was conducted in May 2018. The Census invited participation by:

"...anyone who self-identifies as part of the health information workforce working for/with an organisation that operates in Australia. You are part of the workforce if you work (including 
volunteer or actively seeking) in a role where the primary function is related to developing, maintaining, or governing the systems for the management of health data, health information, or health knowledge" (Butler-Henderson \& Gray, 2018a). Close to 1,600 usable responses were received in the first Census, and it is planned to run it again in 2020, and every three years thereafter. Broad description of the responses is reported elsewhere (Butler-Henderson \& Gray, 2018b). A New Zealand version of the census was conducted in late 2018, auspiced by collaboration with the Australian developers of the tool. It is hoped that further international replications will take place, with the benefit of a common schedule of questions to enable multinational statistical data to be gathered.

We chose a scoping review methodology for this review, as we wished to gain an overview of the size and nature of the literature on a topic that is inherently diffuse, heterogeneous and dynamic. Our review shares the first and third of Paré et al.’s (2015) three possible purposes for this type of review:

"to examine the extent, range and nature of research activities [in a subject]; determine the value of undertaking a full systematic review; or identify research gaps in the extant literature."

\section{Survey methodology}

This protocol reflects the stages for a scoping review, as outlined by Arksey \& O’Malley (2005). We have also followed the PRISMA Extension for Scoping Reviews (PRISMA-ScR) reporting guideline (Tricco et al., 2018).

Research Question. How has the concept of health information work emerged and developed within the wider health sector, information technology or information sciences workforces?

Aim. A thematic and bibliometric literature review to trace the evolution of the idea of health information work.

Objectives. The review has four objectives:

1. To map the published international literature discussing an instrumental or professional human role in health information work from inception to the present, describing its extent, range and nature

2. To provide a review summary of the literature

3. To inform further research

4. To contribute to the current work promoting health information workforce planning, in Australia and internationally.

Definition. For the purpose of this study, "health information work" is defined as human agency in the performance of work with health information. 
Identifying relevant studies. Because of the multidisciplinary nature of health information, a variety of databases are potential sources of the publications to be included in this review. There are multiple databases in each of the three key domains. The resources selected to search were: Ovid Medline (1946 to June 2017), CINAHL Full Text, Embase, Applied Social Sciences Index and Abstracts, and Library, Information Science and Technology Abstracts.

Both authors of this review are health information professionals, with experience developing systematic literature searches. The first author (KG) conceptualized the search, covering the three concepts of health information, management or systems, and work or role or profession. The second author (CG) developed the preliminary search strategy in Ovid Medline using a combination of index terms and keywords. When tested, this strategy yielded over 2,000 results, with a high proportion of irrelevant items. Following discussion, it was agreed to narrow the range of search terms. The final strategy used combinations of keyword searches in title or abstract fields to identify items containing all three concepts:

(health OR healthcare) adjacent to (information OR knowledge OR data) AND informatics OR management OR technology OR library OR systems OR digital AND work* OR profession* OR role* OR staff* OR expert* OR leader* OR champion* OR special*

Once finalized the search was translated to the other bibliographic databases. The searching was performed by two information professionals between July 2017 and March 2018. Each specialist searched a number of the nominated databases; there was no staff cross-over in this searching.

A search for grey literature was also performed, with principal sources being the repository search core.ac.uk and Google Scholar. This yielded fewer than 50 additional relevant English language items. Relevant items were also identified through citation checking.

The final results sets for all database searches were combined, de-duplicated, and exported into the Covidence program to manage the study screening stage.

Study Selection. Items were assessed in two stages, using the pre-determined criteria. Inclusion criteria:

1. Items referring to an instrumental or professional human role in health information work

2. Types of studies: primary research, case studies, reviews, theoretical or analytical studies, policy or planning items

3. Participants: People involved in managing health information, health data, health knowledge; jobs, roles, positions.

Exclusion criteria: 
1. Items describing health information work by consumers, patients, carers (nonprofessional actors)

2. Items describing 'champions' or stakeholders if not recognizing a specialist health information work role

3. Article types: Not editorials, letters

4. Language: Exclude if not in English or no English abstract.

In the first stage, title and abstract screening was performed with each of the two information specialists reviewing half of the citations. Eligible items then passed to the second screening round, which involved review of the article full-text by both information specialists. Discrepancies were resolved through discussion. The search and screening process is shown in the PRISMA diagram (Fig. 1) below.

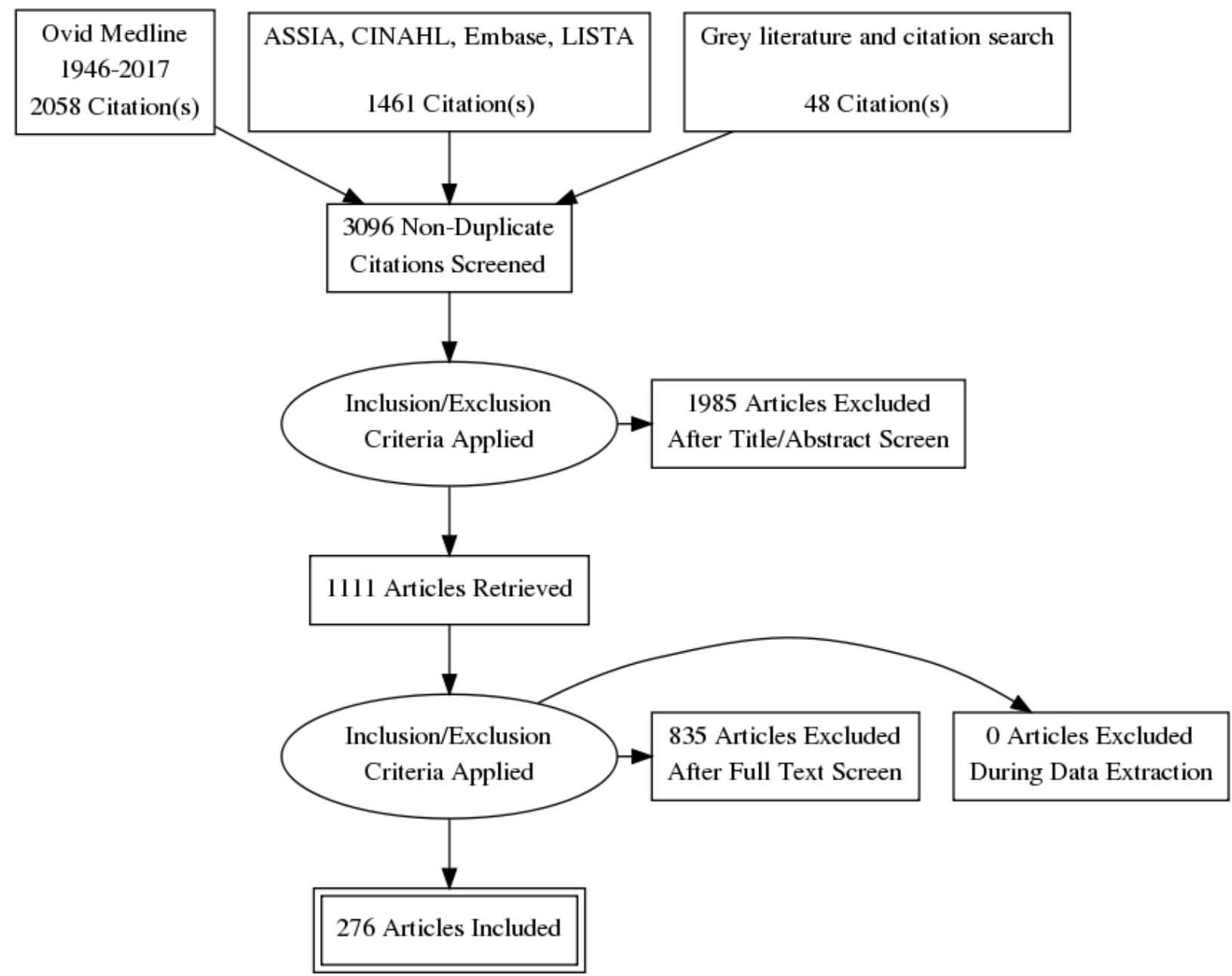

\section{Figure 1: Flow of citations in the Health Information Work scoping review}

For items from the grey literature, two additional criteria were used: 
1. Published by a reputable organisation

2. Contains empirical data about the health information work (i.e. commentary, editorials, or opinion items were not eligible).

Data extraction. A data charting form was devised to standardize extraction of data about each item in the final set. The following characteristics were recorded: year of publication, author(s), country, setting/context, health information position identity, role, responsibilities, functions, knowledge, skills, attributes. Analysis of these features has commenced.

Unlike a systematic review, a scoping review has no requirement to assess the methodological strengths and weaknesses of each included study, nor are the studies assessed for quality or risk of bias (Tricco et al., 2018). We do not aim to report on these characteristics in this review.

Collating, summarizing and reporting results. The review results will give an overview of the evolution and current status of the concept of human agency in health information work. They will include a synthesis of the themes in the included literature, based on deductive analysis related to relevant models of capability or competence (e.g. Sanz et al., 2018, Topi et al., 2017). Results from the data charting will be presented in narrative text, tables and diagrams as appropriate. A descriptive bibliometric analysis will also be undertaken on the publications in the final set. Statistical information will be extracted about the documents in the set, and charts or graphs will be used to display features such as span of publication years and growth rate, prominent authors, authors' geographic distribution, frequently-occurring journals, range of professional identities reported, and other notable characteristics.

\section{Current status of the review}

Preliminary results of the scoping review will be presented in mid-February 2019 at the ITCH Conference (Information Technology \& Communications in Health), University of Victoria, BC, Canada. A detailed report of the review findings will be published in 2019 in an open access publication.

\section{Conclusions}

Major digital health initiatives are being implemented in many countries, at levels from wholeof-hospital to whole-of-health system. Their effects are anticipated to be transformative, and disruptive. An emerging feature of digital health delivery is predicted to be a more fluid health workforce: less routinized, more mobile, more globalized. In contrast, the expertise of a specialist professionalized workforce responsible for the development, maintenance, and governance of the systems for managing health data, health information and health knowledge is considered to be critical to enable safe patient care and health care delivery. This review will contribute to wider research on the present and future health information workforce. 


\section{Acknowledgements}

We acknowledge the valuable assistance of information specialist Simone Pritchard, for her substantial contribution to the searching and screening stages of the review.

\section{References}

Arksey H \& O’Malley L. 2005. Scoping studies: towards a methodological framework. International Journal of Social Research Methodology, 8:19-32. DOI: 10.1080/1364557032000119616.

Butler-Henderson K. \& Gray K. 2018a. Health information workforce census. Available at http://www.utas.edu.au/business-and-economics/hiwcensus (accessed 10 February 2019)

Butler-Henderson K \& Gray K 2018b. Australia's health information workforce: Census summary report 2018. Launceston: University of Tasmania. Available at http://www.utas.edu.au/_data/assets/pdf_file/0003/1163487/Australias-HIW-Census-SummaryReport-2018.pdf (accessed 10 February 2019)

Eardley T. 2006. NHS Informatics workforce survey. London: ASSIST. Available at http://www.bcs.org/upload/pdf/finalreport_20061120102537.pdf (accessed 10 February 2019)

Health Workforce Australia. 2010. Health information workforce report. Available at http://industry.gov.au/Office-of-the-ChiefEconomist/SkilledOccupationList/Documents/2015Submissions/HIMAA-Attachment-4.pdf (accessed 10 February 2019)

Hersh W. 2010. The health information technology workforce: estimations of demands and a framework for requirements. Applied clinical informatics, 1:197-212. DOI:10.4338/ACI-200911-R-0011.

Legg M \& Lovelock B 2009. A review of the Australian health informatics workforce. Melbourne: Health Informatics Society of Australia. Available at https://hisa-siteym.com/resource/resmgr/hisa-resources_page/2009_ahi_workforce-review_v1.pdf (accessed 10 February 2019)

Paré G, Trudel M-C, Jaana M, \& Kitsiou S. 2015. Synthesizing information systems knowledge: A typology of literature reviews. Information \& Management, 52:183-199.

DOI:10.1016/j.im.2014.08.008. 
Sanz LF, Gomez-Perez J, Castillo-Martinez A. 2018. Analysis of the European ICT competence frameworks. In: Ahuja V \& Rathore S. eds. Multidisciplinary perspectives on human capital and information technology professionals. Hershey, PA: IGI Global, 225-245. DOI:10.4018/978-15225-5297-0.

Topi H, Karsten H, Brown SA, Carvalho JA, Donnellan B, Shen J, Tan BC \& Thouin MF. 2017. MSIS 2016 global competency model for graduate degree programs in information systems. Communications of the Association for Information Systems, 40. DOI:10.17705/1CAIS.04018.

Tricco AC, Lillie E, Zarin W, O'Brien KK, Colquhoun H, Levac D, et al. 2018. PRISMA Extension for Scoping Reviews (PRISMA-ScR): Checklist and explanation. Annals of Internal Medicine, 169:467-473. DOI: 10.7326/M18-0850. 


\section{University Library}

\section{- M M I E E R VA A gateway to Melbourne's research publications}

Minerva Access is the Institutional Repository of The University of Melbourne

Author/s:

Gray, K;Gilbert, C

Title:

Health information work - a scoping review protocol

Date:

2019

Citation:

Gray, K. \& Gilbert, C. (2019). Health information work - a scoping review protocol. PeerJ Preprints, https://doi.org/10.7287/peerj.preprints.27535v2.

Persistent Link:

http://hdl.handle.net/11343/247878 